\title{
Teprotumumab Treatment for Thyroid-Associated Ophthalmopathy
}

\author{
Terry J. Smith ${ }^{\mathrm{a}, \mathrm{b}}$

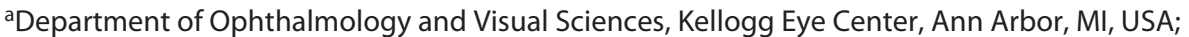 \\ ${ }^{b}$ Division of Metabolism, Endocrinology and Diabetes, Department of Internal Medicine, University of Michigan \\ Medical School, Ann Arbor, MI, USA
}

\section{Keywords}

Graves' disease · Insulin-like growth factor-I receptor ·

Thyroid-associated ophthalmopathy · Autoimmune

\section{Abstract}

Background: Thyroid-associated ophthalmopathy (TAO), an autoimmune process affecting the tissues surrounding the eye, most commonly develops in individuals with Graves' disease. It is disfiguring, can cause vision loss, and dramatically lessens the quality of life in patients. There has been an absence of approved medical therapies for TAO with proven effectiveness and safety in multicenter, placebo-controlled, and adequately powered clinical trials. Summary: The following is a brief overview of the rationale for developing a monoclonal antibody inhibitor of the insulin-like growth factor-I receptor into a treatment for TAO. This area of fundamental research has yielded an effective and safe medication, namely teprotumumab, based on two multicenter, placebo-controlled trials. Teprotumumab, marketed as Tepezza, has been approved recently by the US Food and Drug Administration for the treatment of TAO. Given its remarkable effectiveness, Tepezza is poised to become the first-line standard of care for TAO. Key Messages: Introduction of Tepezza into our armamentarium of therapeutic strategies for TAO represents a paradigm shift in the management of the disease. I proffer that the drug will replace glucocorticoids as a first-line treatment for TAO.

(c) 2020 European Thyroid Association Published by S. Karger AG, Basel

\section{Introduction to Thyroid-Associated Ophthalmopathy}

Graves' disease (GD) and Hashimoto's thyroiditis represent two examples of thyroid autoimmunity with typically contrasting clinical features that can dominate their presentation [1]. GD is frequently manifested as hyperthyroidism while Hashimoto's thyroiditis can result in gland failure. Despite their characteristically differing impact on thyroid function, both diseases can be associated with an activation and remodeling of those tissues surrounding the eye and investing the upper face, a process known as thyroid-associated ophthalmopathy (TAO; also known as thyroid eye disease or Graves' orbitopathy) $[2,3]$. In TAO, the tissues within the orbit become inflamed, congested, and expand, the consequences of targeted immune responses. These immune responses are thought to be intimately related to those occurring in the
Published by S. Karger AG, Basel
Terry J. Smith

Department of Ophthalmology and Visual Sciences Kellogg Eye Center, Brehm Tower, 1000 Wall Street Ann Arbor, MI 48105 (USA)

terrysmi@med.umich.edu 
thyroid. Despite the potential seriousness of TAO, effective treatment of the disease represents an important, unmet need. This void in managing TAO results from its variable presentation and historical lack of preclinical models.

TAO most frequently presents as a variable constellation of signs and symptoms, including eye irritation, increased tearing, eyelid swelling, and lid retraction. It can remain relatively mild or progress to proptosis, diplopia, sight-threatening optic nerve compression and corneal surface breakdown, the consequence of exposure keratopathy. The progression of TAO typically lasts $2-3$ years and gives way to a period when these manifestations cease changing, a temporal pattern first immortalized by Rundle and Wilson [4]. These manifestations can abate spontaneously or remain as factors diminishing ocular function and quality of life. The inflammatory processes occurring during active-phase TAO can culminate in fibrosis of the orbital structures, processes currently presumed to be irreversible without surgical intervention. Those patients with substantial functional and anatomic deficits are commonly subjected to multistaged surgical procedures, which can themselves reactivate TAO [5] or result in suboptimal outcomes. We continue to lack highfidelity discriminators to help identify patients destined to develop serious disease at the early stage of TAO. Moreover, our medicinal tool chest has been devoid of reliably effective agents, especially those capable of mitigating disease severity and altering its outcome.

\section{Currently Available Medical Therapies for TAO Underscore a Glaring Unmet Need}

The therapeutic landscape for active TAO continues to be confronted with the absence of a medical therapy achieving US Food and Drug Administration approval. Until very recently, none had proven safe and effective in adequately powered and placebo-controlled trials [1]. This unmet need has resulted in the widespread use of non-specific agents failing to alter the severity, duration, or outcome of TAO. Glucocorticoids provide a modestly effective reduction of those symptoms resulting from inflammation but only half the patients receiving steroids exhibit a clear-cut benefit [6]. Even among those patients responding, clinical improvement is largely limited to reduction in signs and symptoms attributable to inflammation, such as redness, swelling, and ocular discomfort. No persuasive evidence has been generated demonstrating that these agents alter the disease course. Among the most carefully conducted studies of steroids is that of Bartalena et al. [7], who administered by infusion three different dosages of methylprednisolone $(2.25,4.98$, and $7.47 \mathrm{~g})$. The study patients received 12 weekly infusions after which clinical activity scores (CAS) were determined. Those subjects who received $7.47 \mathrm{~g}$ derived the greatest responses, although these were not durable. A proptosis reduction of $0.6 \mathrm{~mm}$ compared to baseline was detected in that cohort, considered as falling below clinical significance. In practice, no one administration protocol is used by all care providers; methylprednisolone is frequently given over a 12 -week course of $500 \mathrm{mg}$ per week for the first 6 doses and $250 \mathrm{mg}$ per week for the remainder [8]. Importantly, wide experience with steroid treatment strongly suggests that neither proptosis nor diplopia is convincingly affected by steroid treatment, mirroring the aforementioned trial result [9]. The side effect profile of high-dose glucocorticoid infusions concerns the potential for severe hepatic toxicity $[10,11]$. Thus, despite their frequent use, glucocorticoids are of limited therapeutic value and are fraught with substantial side effects. Despite these shortcomings, many experts in the field express reticence to venture beyond their use in TAO, especially as "first-line" therapy [12].

\section{Insight into the Biological Underpinnings of TAO Has Allowed Examination of Targeted Therapies}

The more recent introduction of biologics to the medley of agents in our medicinal armamentarium for other autoimmune diseases has prompted limited repurposing to TAO. The most extensively studied is rituximab, an anti-CD20 monoclonal antibody that results in the destruction of B-lymphocytes [13]. Rituximab was initially developed for non-Hodgkin's lymphoma but subsequently became useful for treating rheumatoid arthritis [14], scleroderma, systemic lupus erythematosus, and other autoimmune diseases [15-17]. A number of laboratory groups, including that of Hasselbalch [18], were among the first to consider rituximab in GD and TAO. A number of small series examining the drug had appeared $[19,20]$, but it remained for two contemporaneously performed studies at separate institutions to meaningfully test the efficacy of rituximab in TAO. One, conducted at Mayo Clinic, Rochester, was placebo controlled, comparing subjects treated with rituximab to those receiving saline [21]. It failed to disclose differences between the active drug and placebo in reducing CAS. The other, conducted in Milan, Italy, compared rituximab to meth-
Smith 
Theoretical Model of the Pathogenesis of Thyroid-Associated Ophthalmopathy

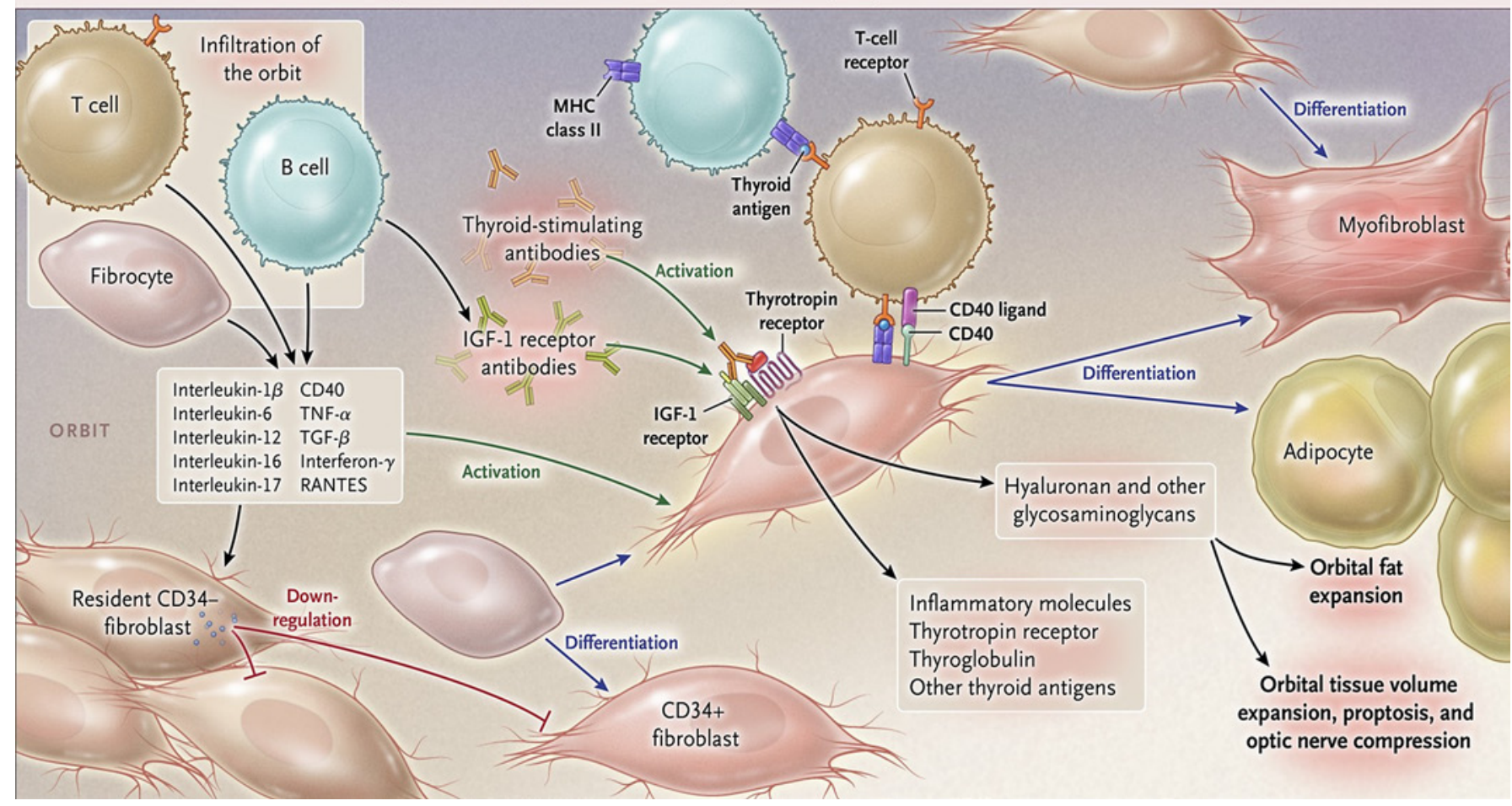

Fig. 1. Theoretical model of the pathogenesis of TAO. CD34+ fibrocytes, monocyte-derived progenitor cells originating from the bone marrow, circulate in patients with GD at higher abundance than in healthy individuals. Fibrocytes promiscuously express the TSHR, thyroglobulin, thyroperoxidase, and sodium-iodide symporter. They also constitutively express class II major histocompatibility complex (MHC) and are capable of presenting antigens to T cells. Fibrocytes have the capacity to differentiate into CD34+ fibroblasts, myofibroblasts, and triglyceride-accumulating adipocytes. Several thyroid-related genes expressed by fibrocytes can be detected at considerably lower levels in CD34+ fibroblasts. When activated, CD34+ fibroblasts, which reside as an admixture with CD34- fibroblasts, generate several proinflammatory or anti-in-

ylprednisolone and found that the biological drug was more effective in reducing clinical activity [22]. Neither trial of rituximab yielded evidence that anti-CD20 targeting of $\mathrm{B}$ cells was effective in reducing the proptosis or diplopia of TAO.

Other agents have been examined in the recent past, most already used for other autoimmune diseases. A combination of methylprednisolone and mycophenolate was compared to treatment with methylprednisolone alone [23]. A total of 164 patients with moderate to severe active TAO were subjected to the study which failed to convincingly demonstrate the benefit of adding mycophenolate to the steroid alone. No differences emerged in response rate at 12 weeks or relapse rate at 24 and 36 flammatory cytokines, including IL- $1 \beta, 6,8,10,12,16$, TNF- $\alpha$, and regulated on activation, normal $\mathrm{T}$ cell expressed and secreted (RANTES), CXCL-12, and CD154, as well as receptors for certain cytokines (examples are CXCR4 and CD40). The surface of orbital fibroblasts displays IGF-IR. These fibroblasts express three mammalian hyaluronan synthase isoenzymes and UDP glucose dehydrogenase and synthesize hyaluronan, the glycosaminoglycan associated with expanding orbital tissue in TAO. Many of these phenotypic attributes exhibited by CD34+ orbital fibroblasts appear to be downregulated by the neural axon repellent, Slit2, which is synthesized and released by CD34- orbital fibroblasts. (From Smith and Hegedüs [1], ${ }^{\circ}$ Massachusetts Medical Society, reprinted with permission.)

weeks. The study data were subjected to post hoc analysis which suggested that mycophenolate in addition to methylprednisolone improved the response rate at 24 weeks when compared to steroid alone.

Several biological agents targeting specific cytokines have also been preliminarily examined in TAO. The rationale for this consideration may have emanated from basic observations related to the disease mechanism. Abnormalities in serum tumor necrosis factor- $\alpha$ (TNF- $\alpha$ ) levels and those of IL- 6 were detected in patients with TAO [24]. Moreover, immunoreactivity to TNF- $\alpha$ had been detected in affected orbital tissues [25]. In addition, orbital fibroblasts and fibrocytes from patients with TAO were found to generate several cytokines following their 
activation by TSH, TSIs, and insulin-like growth factor-I (IGF-I), including IL-6, IL-1 $\beta$, IL-1 receptor antagonist, IL-8, IL-12, IL-16, IL-23, and CD40, and regulated on activation, normal $\mathrm{T}$ cell expressed and secreted (RANTES, CCL5) [26-31]. Therapy with infliximab and etanercept, anti-TNF- $\alpha$ monoclonal antibodies, has been described in patients with TAO $[32,33]$. The limited numbers of patients treated with anti-TNF- $\alpha$ agents and lack of controls makes any meaningful judgements impossible at this juncture. The IL-6 receptor-inhibiting antibody, tocilizumab, has been studied in patients with steroid-resistant TAO in a single institution [34].

\section{Implication of the IGF-I Receptor in the Pathogenesis of TAO Has Led to the Development of Teprotumumab as a Medical Therapy}

The concept of interfering with IGF-I receptor (IGFIR) signaling as a therapeutic approach to human disease evolved from a growing understanding of how the receptor and its pathways regulate mammalian physiology in health and in disease (Fig. 1). The protein was linked to the process of neoplastic cell transformation 30 years ago [35]. Involvement of IGF-IR in the development and progression of some cancers [36] had resulted in the initiation of several therapy-developmental programs, each at a separate pharmaceutical company, focusing on inhibitors of IGF-IR [37]. As a consequence of the generally ineffective profiles of all drugs under development, including both monoclonal antibodies and small molecules, each tested in a variety of different tumor types, these programs were largely curtailed. Thus, one of the previously developed agents, a monoclonal IGF-IR inhibitory antibody, designated R1507, became available for repurposing to other diseases. Despite its poor efficacy in treating tumors, the drug exhibited a favorable safety profile, thus making it an ideal candidate for TAO.

The IGF-IR pathway appears to regulate immune function and has been insinuated in the pathogenesis of autoimmune disease [38]. A potential nexus between IGF-IR and GD emerged from the early observations of Weightman et al. [39], who demonstrated that immunoglobulin G from patients with the disease (GD-IgG) could displace radiolabeled IGF-I from binding sites displayed on the surface of orbital fibroblasts. The findings of Ingbar and colleagues [40] in the 1980s demonstrated the synergistic effects of IGF-I and insulin on TSH and GDIgG actions in cultured clonal thyroid cells. These observations set the table for better understanding the molecu- lar and functional interactions between thyrotropin receptor (TSHR) and IGF-IR. Subsequently, Pritchard et al. [26] demonstrated that GD-IgG could induce cytokine and chemokine-encoding mRNAs that are involved in $\mathrm{T}$ cell recruitment to inflamed tissues. These inductions involve the IGF-IR since either knocking down the receptor or inhibiting its activity could attenuate cytokine inductions. The actions of GD-IgG are also mediated through the activation of the Akt/FRAP/mTOR/p70 ${ }^{\text {S6k }}$ pathway [41]. The finding of autoantibodies directed at IGF-IR in patients with GD raise a question that remains unanswered - are they active and do their interactions with IGF-IR initiate signaling independent of the actions occurring directly on the TSHR? Evidence has been reported for either supporting signal-initiating IGF-IR-targeting autoantibodies $[26,41-44]$ or failing to detect them $[45,46]$. Recognition of IGF-IR and TSHR colocalization and the dependence of signaling downstream from TSHR on IGF-IR activity provided additional, critical insights [47]. These findings underpinned a strong rationale for examining what clinical benefit, if any, interrupting the IGF-IR signaling pathway might have in treating TAO.

\section{Insight into IGF-IR Involvement in TAO Led to Two Clinical Trials of Teprotumumab}

\section{Initial Phase 2 Trial}

Armed with results of many studies, almost entirely conducted in vitro nearly 2 decades ago, we set out to directly test the hypothesis that interrupting the IGF-IR pathway would represent an effective therapeutic strategy for TAO. A multicenter, placebo-controlled, phase 2 trial of teprotumumab was organized, starting in 2010 in collaboration with its sponsor, River Vision Development, which provided financial support [48]. Its design and the creation of a study protocol resulted from this academic/commercial collaboration which was standardized across all performance sites. A total of 15 sites across North America and Western Europe recruited patients between July 2, 2013 and September 23, 2015. Individuals between the ages of 18 and 75 years whose TAO was very active as defined by a CAS of $\geq 4$ points on a 7-point scale, and who had developed TAO $\leq 9$ months prior to study enrollment were included. Participants needed to be close to chemically euthyroid (serum FT4 and FT3 were within $50 \%$ above or below the upper and lower normal limits of the assays used). Female patients were required to have negative pregnancy testing at baseline and used approved contraception for the duration of the treatment phase. Patients having under- 
gone surgical correction of their TAO in the past, those who had received rituximab, and those who had a history of glucocorticoid therapy for their TAO with a cumulative dose of $>1 \mathrm{~g}$ of methylprednisolone (or equivalent) were excluded. The trial comprised three phases: screening, intervention (treatment), and a 48-week follow-up. Following screening, patients were assessed at baseline and every 3 weeks at the time they underwent infusion with either placebo and teprotumumab. These clinical examinations were routinely conducted by the same ophthalmologist and proptosis was quantified using the same Hertel instrument.

In total, 88 eligible patients meeting study criteria were randomly assigned to either of two double-masked treatment arms at a ratio of $1: 1$. A total of 87 patients were included in the intention to treat group (45 in the placebo arm and 42 receiving the active drug). Participants were stratified at each trial center with regard to smoking history. The primary response was defined as a reduction of $\geq 2$ points in the 7 -item CAS scale and a reduction in proptosis of $\geq 2 \mathrm{~mm}$ at week 24 in the more severely affected (study) eye without similar worsening in the fellow (contralateral) eye. Any patient lacking 24-week data was judged a treatment failure. Secondary endpoints included proptosis, CAS, diplopia, and quality of life (using a GO-QoL score [49]), which were assessed using a model of repeated measurements and expressed as change from baseline. Responses in the non-study eye were analyzed identically to those in the study eye.

Overall, 39 patients (87\%) receiving placebo and 37 (88\%) receiving teprotumumab completed the intervention phase of the study. Attempts to stratify both treatment cohorts with regard to smoking status were unsuccessful in that an imbalance was detected with greater representation of smokers in the placebo group. While the baseline characteristics of the two treatment groups were nearly identical, 29 out of 42 (69\%) patients in the teprotumumab cohort responded (i.e., experienced a $\geq 2$-point reduction in CAS and a $\geq 2-\mathrm{mm}$ reduction in proptosis in the study eye), compared to 9 out of 45 (20\%) of those receiving placebo met the primary response $(p<0.001)$. In the per-protocol cohort, 8 out of $36(22 \%)$ patients receiving placebo and 26 out of 33 (79\%) patients receiving teprotumumab met the primary response at 24 weeks $(p<$ $0.001)$. Teprotumumab shortened the interval from baseline for achieving a primary response; the impact of the drug was rapid and differed from placebo at 6,12 , and 18 weeks $(p<0.001)$. Patients achieving an improvement of $\geq 3$ points in CAS and $\geq 3 \mathrm{~mm}$ in proptosis reduction were more numerous in the teprotumumab group than in the placebo cohort $(p<0.001)$. With regard to secondary re-

Teprotumumab and Thyroid-Associated Ophthalmopathy sponses, measured as continuous variables from baseline, both CAS and proptosis reductions were greater in those treated with teprotumumab than individuals in the placebo group at every time point ( $p<0.001$ for all comparisons). Furthermore, the number of patients achieving a reduction of proptosis $\geq 4 \mathrm{~mm}$ was 17 out of $42(40 \%)$ of those treated with teprotumumab compared to $0 \%$ receiving placebo. Visual-scale GO-QoL increased to a greater extent in the teprotumumab group compared to placebo at every time point. In contrast, the appearance subscale, while consistently improving as a function of treatment duration, failed to achieve statistical significance. Subjective diplopia improved and was significantly greater at every time point in those receiving teprotumumab $(p<0.001$ at week 24). The overall safety profile of the drug appeared to be favorable in the trial. Among the adverse events was hyperglycemia, occurring largely in individuals diagnosed with diabetes mellitus before their trial participation. These were generally managed by increasing anti-hyperglycemia medications. Importantly, the worsening of glycemic control was transient and requirement for diabetes medication returned to baseline following the intervention phase of the study. Other adverse events associated with teprotumumab included hearing abnormalities and muscle cramps. These too were transient. Despite attempts to balance tobacco use in the two treatment groups, more smokers were included in the placebo cohort.

\section{Pivotal Phase 3 Trial Confirming the Results of the Earlier Study}

A phase 3 pivotal trial, conducted at 13 sites in North America and Europe, enrolled patients between October 24, 2017 and August 31, 2018 [50]. The study included 83 patients with active, moderate to severe TAO who were randomly assigned to either the placebo $(n=42)$ or teprotumumab $(n=41)$ group. As in the phase 2 trial, the study was placebo controlled, double masked, and all patients exhibited moderate to severe TAO of $\leq 9$ month's duration, were close to euthyroid, and had not undergone orbital surgery, radiotherapy, or received immunomodulatory therapy with the exception of $<1 \mathrm{~g}$ of prednisone (following a 6-week washout). In contrast to the initial trial, a slightly broadened age range was allowed and the primary outcome was a reduction in proptosis $\geq 2 \mathrm{~mm}$ in the study eye at week 24 . Secondary outcomes included an aggregate of reduction in CAS $\geq 2$ points and improvement in proptosis $\geq 2 \mathrm{~mm}$, achieving a CAS of 0 or 1 , mean change in proptosis across trial visits, reduction in diplopia $>1$ grade, and mean change in overall score in the GO-QoL questionnaire from baseline to week 24 . The 

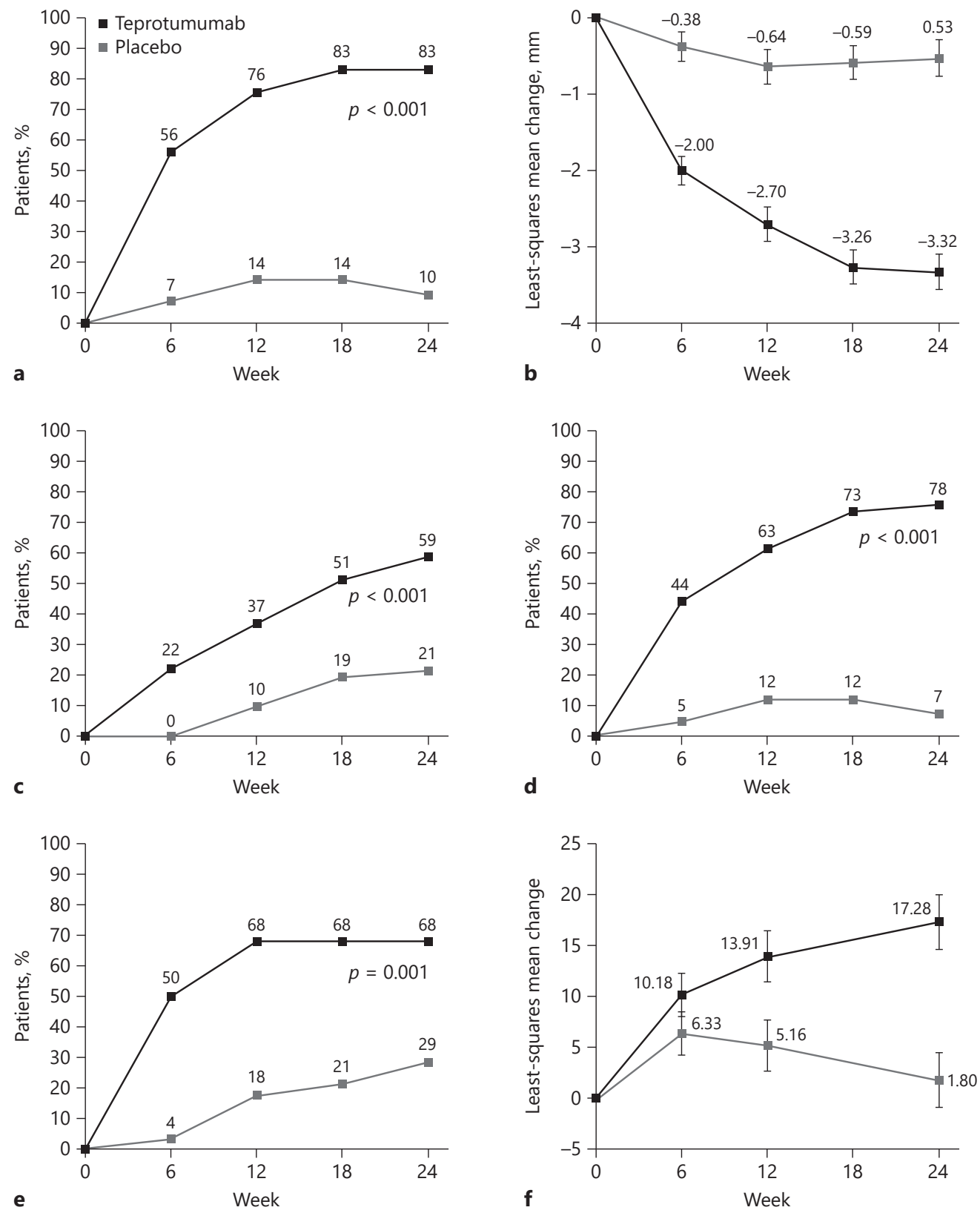

Fig. 2. Efficacy outcomes over the phase 3 trial; 41 patients were randomized to the teprotumumab treatment group and 42 to the placebo cohort. a The primary outcome was the percentage of patients with a proptosis response ( $\geq 2 \mathrm{~mm}$ proptosis reduction) in the study without a similar worsening in the fellow (contralateral) eye. b Change from baseline in proptosis ( \pm standard error). c Percentage of patients with a CAS of 0 or 1 at week 24 . d Percentage of patients with an overall response. e Percentage of patients with diplopia response. $\mathbf{f}$ Change from baseline in the overall GO-QOL questionnaire score. (From Douglas et al. [50], ${ }^{\complement}$ Massachusetts Medical Society, reprinted with permission.) 


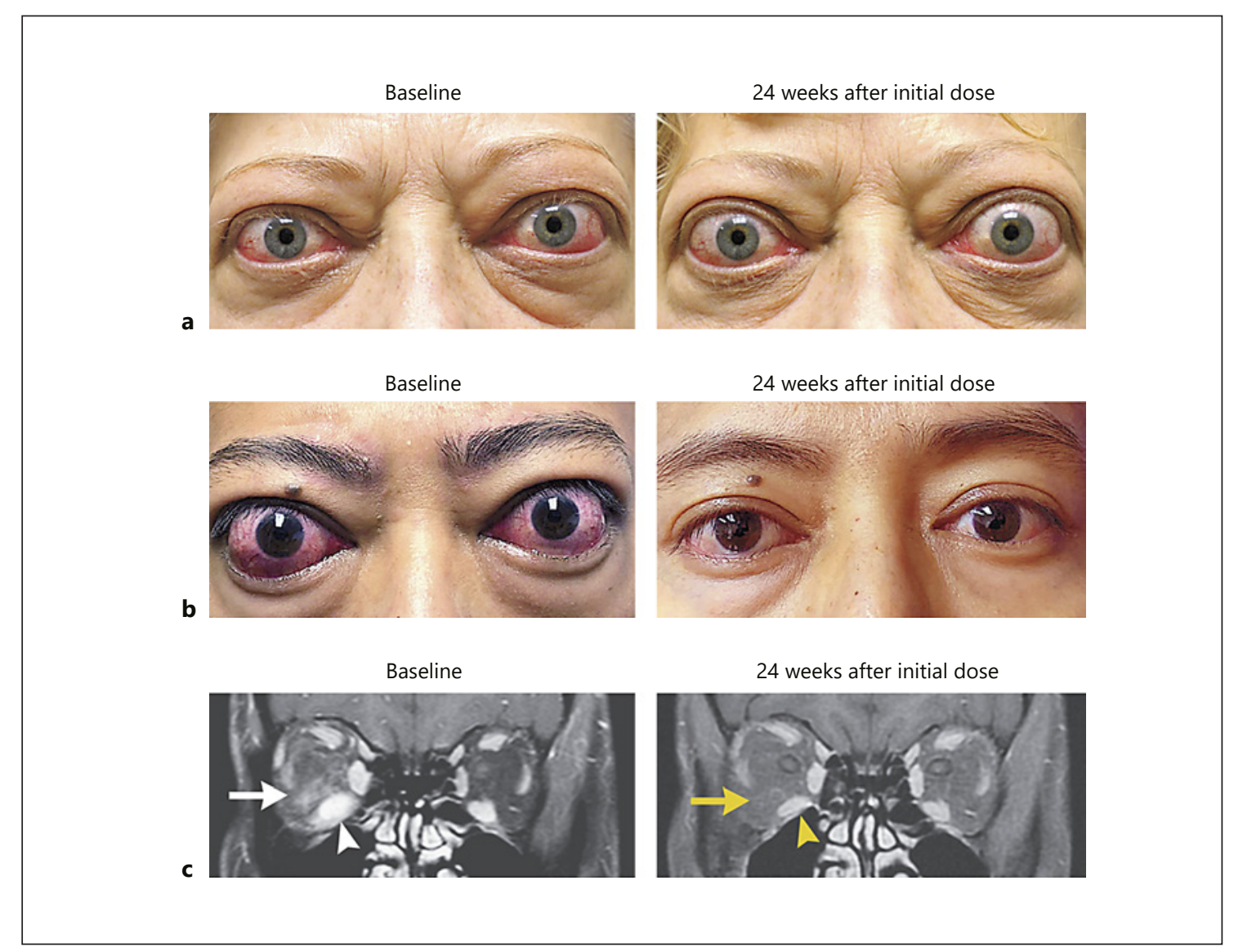

Fig. 3. Facial images and MRIs at baseline and 24 weeks following therapy initiation. a Photographic image at baseline of a patient receiving placebo who exhibited proptosis (left $29 \mathrm{~mm}$ and right 27 $\mathrm{mm}$ ), edema, and several inflammatory signs (left CAS 7 and right CAS 5). Considerable proptosis (left $28 \mathrm{~mm}$ and right $26 \mathrm{~mm}$ ) and inflammatory signs (left CAS 5 and right CAS 5) remained at week 24. b Photographic image at baseline of a patient receiving teprotumumab with baseline proptosis (bilateral $24 \mathrm{~mm}$ ), upper and lower eyelid retraction, edema, and several inflammatory signs (bilateral CAS 5). In contrast, reductions in proptosis $(-5 \mathrm{~mm})$ and CAS $(-4$ points) were achieved in both eyes following treatment. c Coronal, contrast-enhanced, fat-saturated, T1-weighted MRIs from a pa-

percentage of patients with a proptosis reduction $\geq 2 \mathrm{~mm}$ was greater at week 24 in those receiving teprotumumab (83\%) than in the placebo controls $(10 \% ; p<0.001$; Fig. 2$)$. The number needed to treat was 1.36 . All secondary outcomes were better in the patients receiving teprotumumab. Substantial orbital fat and extraocular muscle volume reductions were detected in the 6 patients undergoing orbital imaging at baseline and at week 24 (Fig. 3). Longterm follow-up of patients revealed that $56 \%$ of week 24 proptosis responders and $58 \%$ of week 24 diplopia responders maintained their responses at week 72 (unpub-

Teprotumumab and Thyroid-Associated Ophthalmopathy tient receiving teprotumumab. Baseline MRI demonstrates marked inferior rectus muscle (white arrowhead) and orbital fat (white arrow) enhancements and enlarged inferior rectus muscle (white arrowhead). Proptosis was $23 \mathrm{~mm}$, CAS 5, and Gorman subjective diplopia score 3 , which denotes continuous diplopia in the primary gaze. MRI demonstrates resolution of enhancement of inferior rectus muscle (yellow arrowhead) and orbital fat (yellow arrow) and inferior rectus muscle volume ( $-49 \%$, yellow arrowhead) and medial rectus muscle volume reduction $(-41 \%)$ at week 24 . These changes coincided with proptosis of $18 \mathrm{~mm}$, CAS 0 , and Gorman subjective diplopia score 0. (From Douglas et al. [50], ${ }^{\odot}$ Massachusetts Medical Society, reprinted with permission.)

lished observations). No additional safety signals were identified in this follow-up study and serious adverse events were infrequent. Unlike the phase 2 study, smokers were balanced in treatment groups in the phase 3 trial.

\section{Conclusion}

More detailed characterization of the pathways involved in the pathogenesis of TAO, including the unanticipated role of IGF-IR in this disease process, has led 
to the development of teprotumumab, a monoclonal antibody inhibitor of that tyrosine kinase receptor as therapy in the disease. The drug has been approved recently by the US Food and Drug Administration as the first medical therapy for this disfiguring and potentially blinding disease. Registration followed the successful conclusion of 2 clinical trials which demonstrated remarkable effectiveness in reversing disease severity and attenuating clinical activity. The safety profile of teprotumumab is extremely favorable, with the majority of adverse events judged as mild. Thus, the drug is poised to become the first-line therapy for TAO. It is possible that its use may obviate, at least in some cases, the need for surgical remediation to restore both form and function. Its substantial financial costs must be weighed against that of traditional therapies, including other biological agents and surgeries which are frequently multi-staged.

\section{Acknowledgements}

The author is grateful for the expert assistance in preparing this manuscript from Ms. Leslie Bordine and Ms. Dana Barnhart.

\section{Statement of Ethics}

This work was conducted in accordance with the ethical policies of the University of Michigan Health System.

\section{Disclosure Statement}

T.J.S. has been issued US patents covering his inventions concerning the use of IGF-IR inhibitors as therapy in Graves' disease and TAO. These patents are held by UCLA School of Medicine and Los Angeles Biomedical Research Institute (now Lundquist Institute at Harbor/UCLA). He is a paid consultant for Horizon Therapeutics and Immunovan Corporation.

\section{Funding Sources}

This work was supported in part by National Institutes of Health grants EY008976, EY11708, DK063121, 5UMIA110557, a Core Center for Research grant EY007003 from the NEI, an unrestricted grant from the Research to Prevent Blindness, and by the Bell Charitable Family Foundation.

\section{Author Contributions}

T.J.S. wrote the paper and conducted the background research.

\section{References}

1 Smith TJ, Hegedüs L. Graves' Disease. N Engl J Med. 2016 Oct;375(16):1552-65.

2 Bahn RS. Graves' ophthalmopathy. N Engl J Med. 2010 Feb;362(8):726-38.

3 Wang Y, Smith TJ. Current concepts in the molecular pathogenesis of thyroid-associated ophthalmopathy. Invest Ophthalmol Vis Sci. 2014 Mar;55(3):1735-48.

4 Rundle FF, Wilson CW. Development and course of exophthalmos and ophthalmoplegia in Graves' disease with special reference to the effect of thyroidectomy. Clin Sci. 1945;5(3-4): 177-94.

5 Baldeschi L, Lupetti A, Vu P, Wakelkamp IM, Prummel MF, Wiersinga WM. Reactivation of Graves' orbitopathy after rehabilitative orbital decompression. Ophthalmology. 2007 Jul;114(7):1395-402.

6 Bartalena L, Baldeschi L, Boboridis K, Eckstein A, Kahaly GJ, Marcocci C, et al.; European Group on Graves' Orbitopathy (EUGOGO). The 2016 European Thyroid Association/European Group on Graves' Orbitopathy Guidelines for the Management of Graves' Orbitopathy. Eur Thyroid J. 2016 Mar;5(1): 9-26.

7 Bartalena L, Krassas GE, Wiersinga W, Marcocci C, Salvi M, Daumerie C, et al.; European Group on Graves' Orbitopathy. Efficacy and safety of three different cumulative doses of intravenous methylprednisolone for moderate to severe and active Graves' orbitopathy. J Clin Endocrinol Metab. 2012 Dec;97(12): 4454-63.

8 Kahaly GJ, Pitz S, Hommel G, Dittmar M. Randomized, single blind trial of intravenous versus oral steroid monotherapy in Graves' orbitopathy. J Clin Endocrinol Metab. 2005 Sep;90(9):5234-40.

9 Bartalena L. Prevention of Graves' ophthalmopathy. Best Pract Res Clin Endocrinol Metab. 2012 Jun;26(3):371-9.

10 Le Moli R, Baldeschi L, Saeed P, Regensburg N, Mourits MP, Wiersinga WM. Determinants of liver damage associated with intravenous methylprednisolone pulse therapy in Graves' ophthalmopathy. Thyroid. 2007 Apr; 17(4):357-62

11 Moleti M, Giuffrida G, Sturniolo G, Squadrito $\mathrm{G}$, Campennì A, Morelli S, et al. Acute liver damage following intravenous glucocorticoid treatment for Graves' ophthalmopathy. Endocrine. 2016 Oct;54(1):259-68.

12 Smith TJ, Bartalena L. Will biological agents supplant systemic glucocorticoids as the firstline treatment for thyroid-associated ophthalmopathy? Eur J Endocrinol. 2019 Nov; 181(5):D27-43

13 Maloney DG, Grillo-López AJ, White CA Bodkin D, Schilder RJ, Neidhart JA, et al.
IDEC-C2B8 (Rituximab) anti-CD20 monoclonal antibody therapy in patients with relapsed low-grade non-Hodgkin's lymphoma. Blood. 1997 Sep;90(6):2188-95.

14 Scott DL, Wolfe F, Huizinga TW. Rheumatoid arthritis. Lancet. 2010 Sep;376(9746): 1094-108.

15 Edwards JC, Leandro MJ, Cambridge G. Blymphocyte depletion therapy in rheumatoid arthritis and other autoimmune disorders. Biochem Soc Trans. 2002 Aug;30(4): 824-8.

16 Silverman GJ, Weisman S. Rituximab therapy and autoimmune disorders: prospects for anti-B cell therapy. Arthritis Rheum. 2003 Jun; 48(6):1484-92.

17 Eisenberg R, Albert D. B-cell targeted therapies in rheumatoid arthritis and systemic lupus erythematosus. Nat Clin Pract Rheumatol. 2006 Jan;2(1):20-7.

18 Hasselbalch HC. B-cell depletion with rituximab-a targeted therapy for Graves' disease and autoimmune thyroiditis. Immunol Lett. 2003 Jul;88(1):85-6

19 El Fassi D, Nielsen CH, Hasselbalch HC, Hegedüs $\mathrm{L}$. The rationale for $\mathrm{B}$ lymphocyte depletion in Graves' disease. Monoclonal antiCD20 antibody therapy as a novel treatment option. Eur J Endocrinol. 2006 May;154(5): 623-32. 
20 El Fassi D, Nielsen CH, Hasselbalch HC, Hegedüs L. Treatment-resistant severe, active Graves' ophthalmopathy successfully treated with B lymphocyte depletion. Thyroid. 2006 Jul;16(7):709-10.

21 Stan MN, Garrity JA, Carranza Leon BG, Prabin T, Bradley EA, Bahn RS. Randomized controlled trial of rituximab in patients with Graves' orbitopathy. J Clin Endocrinol Metab. 2015 Feb;100(2):432-41.

22 Salvi M, Vannucchi G, Currò N, Campi I, Covelli D, Dazzi D, et al. Efficacy of B-cell targeted therapy with rituximab in patients with active moderate to severe Graves' orbitopathy: a randomized controlled study. J Clin Endocrinol Metab. 2015 Feb;100(2):422-31.

23 Kahaly GJ, Riedl M, König J, Pitz S, Ponto K, Diana T, et al.; European Group on Graves' Orbitopathy (EUGOGO). Mycophenolate plus methylprednisolone versus methylprednisolone alone in active, moderate-to-severe Graves' orbitopathy (MINGO): a randomised, observer-masked, multicentre trial. Lancet Diabetes Endocrinol. 2018 Apr;6(4): 287-98.

24 Wakelkamp IM, Gerding MN, Van Der Meer JW, Prummel MF, Wiersinga WM. Both Th1and Th2-derived cytokines in serum are elevated in Graves' ophthalmopathy. Clin Exp Immunol. 2000 Sep;121(3):453-7.

25 Heufelder AE, Bahn RS. Detection and localization of cytokine immunoreactivity in retro-ocular connective tissue in Graves' ophthalmopathy. Eur J Clin Invest. 1993 Jan; 23(1):10-7.

26 Pritchard J, Han R, Horst N, Cruikshank WW, Smith TJ. Immunoglobulin activation of $\mathrm{T}$ cell chemoattractant expression in fibroblasts from patients with Graves' disease is mediated through the insulin-like growth factor I receptor pathway. J Immunol. 2003;170: 6348-54.

27 Raychaudhuri N, Fernando R, Smith TJ. Thyrotropin regulates IL-6 expression in CD34+ fibrocytes: clear delineation of its cAMP-independent actions. PLoS One. 2013 Sep; 8(9):e75100.

$28 \mathrm{Li} \mathrm{B}$, Smith TJ. PI3K/AKT pathway mediates induction of IL-1RA by TSH in fibrocytes: modulation by PTEN. J Clin Endocrinol Metab. 2014 Sep;99(9):3363-72.

29 Mester T, Raychaudhuri N, Gillespie EF, Chen H, Smith TJ, Douglas RS. CD40 expression in fibrocytes is induced by TSH: potential synergistic immune activation. PLoS One. 2016 Sep;11(9):e0162994.
30 Fernando R, Adkins SJ, Smith TJ. Slit2 may underlie divergent induction by thyrotropin of IL-23 and IL-12 in human fibrocytes. J Immunol. 2020;204:1724-1735.

31 Wakelkamp IM, Bakker O, Baldeschi L, Wiersinga WM, Prummel MF. TSH-R expression and cytokine profile in orbital tissue of active vs. inactive Graves' ophthalmopathy patients. Clin Endocrinol (Oxf). 2003 Mar; 58(3):280-7.

32 Durrani OM, Reuser TQ, Murray PI. Infliximab: a novel treatment for sight-threatening thyroid associated ophthalmopathy. Orbit. 2005 Jun;24(2):117-9.

33 Paridaens D, van den Bosch WA, van der Loos TL, Krenning EP, van Hagen PM. The effect of etanercept on Graves' ophthalmopathy: a pilot study. Eye (Lond). 2005 Dec; 19(12):1286-9.

34 Perez-Moreiras JV, Gomez-Reino JJ, Maneiro JR, Perez-Pampin E, Romo Lopez A, Rodríguez Alvarez FM, et al.; Tocilizumab in Graves Orbitopathy Study Group. Efficacy of tocilizumab in patients with moderate-to-severe corticosteroid-resistant Graves orbitopathy: a randomized clinical trial. Am J Ophthalmol. 2018 Nov; 195:181-90.

35 Kaleko M, Rutter WJ, Miller AD. Overexpression of the human insulinlike growth factor I receptor promotes ligand-dependent neoplastic transformation. Mol Cell Biol. 1990 Feb;10(2):464-73.

36 Mauro L, Naimo GD, Ricchio E, Panno ML, Andò S. Cross-talk between adiponectin and IGF-IR in breast cancer. Front Oncol. 2015 Jul;5:157.

37 Qu X, Wu Z, Dong W, Zhang T, Wang L, Pang Z, et al. Update of IGF-1 receptor inhibitor (ganitumab, dalotuzumab, cixutumumab, teprotumumab and figitumumab) effects on cancer therapy. Oncotarget. 2017 Apr;8(17):29501-18.

38 Smith TJ. Insulin-like growth factor-I regulation of immune function: a potential therapeutic target in autoimmune diseases? Pharmacol Rev. 2010 Jun;62(2):199-236.

39 Weightman DR, Perros P, Sherif IH, KendallTaylor P. Autoantibodies to IGF-1 binding sites in thyroid associated ophthalmopathy. Autoimmunity. 1993;16(4):251-7.

40 Tramontano D, Cushing GW, Moses AC, Ingbar SH. Insulin-like growth factor-I stimulates the growth of rat thyroid cells in culture and synergizes the stimulation of DNA synthesis induced by TSH and Graves'-IgG. Endocrinology. 1986 Aug;119(2):940-2.
41 Pritchard J, Horst N, Cruikshank W, Smith TJ. Igs from patients with Graves' disease induce the expression of $\mathrm{T}$ cell chemoattractants in their fibroblasts. J Immunol. 2002; 168:942-50.

42 Smith TJ, Hoa N. Immunoglobulins from patients with Graves' disease induce hyaluronan synthesis in their orbital fibroblasts through the self-antigen, insulin-like growth factor-I receptor. J Clin Endocrinol Metab. 2004 Oct; 89(10):5076-80.

43 Gianoukakis AG, Douglas RS, King CS, Cruikshank WW, Smith TJ. Immunoglobulin G from patients with Graves' disease induces interleukin-16 and RANTES expression in cultured human thyrocytes: a putative mechanism for T-cell infiltration of the thyroid in autoimmune disease. Endocrinology. 2006 Apr;147(4):1941-9.

44 Varewijck AJ, Boelen A, Lamberts SW, Fliers E, Hofland LJ, Wiersinga WM, et al. Circulating IgGs may modulate IGF-I receptor stimulating activity in a subset of patients with Graves' ophthalmopathy. J Clin Endocrinol Metab. 2013 Feb;98(2):769-76.

45 Minich WB, Dehina N, Welsink T, Schwiebert C, Morgenthaler NG, Köhrle J, et al. Autoantibodies to the IGF1 receptor in Graves' orbitopathy. J Clin Endocrinol Metab. 2013 Feb; 98(2):752-60.

46 Krieger CC, Neumann S, Marcus-Samuels B, Gershengorn MC. TSHR/IGF-1R cross-talk, not IGF-1R stimulating antibodies, mediates Graves' ophthalmopathy pathogenesis. Thyroid. 2017 May;27(5):746-7.

47 Tsui S, Naik V, Hoa N, Hwang CJ, Afifiyan NF, Sinha Hikim A, Gianoukakis AG, Douglas RS, Smith TJ. Evidence for an association between thyroid-stimulating hormone and insulin-like growth factor 1 receptors: a tale of two antigens implicated in Graves' disease. J Immunol. 2008;181:4397-405.

48 Smith TJ, Kahaly GJ, Ezra DG, Fleming JC, Dailey RA, Tang RA, et al. Teprotumumab for thyroid-associated ophthalmopathy. N Engl J Med. 2017 May;376(18):1748-61.

49 Terwee CB, Dekker FW, Mourits MP, Gerding MN, Baldeschi L, Kalmann R, et al. Interpretation and validity of changes in scores on the Graves' ophthalmopathy quality of life questionnaire (GO-QOL) after different treatments. Clin Endocrinol. 2001 Mar;54(3): 391-8.

50 Douglas RS, Kahaly GJ, Patel A, Sile S, Thompson EH, Perdok R, et al. Teprotumumab for the treatment of active thyroid eye disease. N Engl J Med. 2020 Jan;382(4):34152. 